\title{
Directed forgetting effects in pigeons: Remember cues initiate rehearsal
}

\author{
ANGELO SANTI and JULIE SAVICH \\ Wilfrid Laurier University, Waterloo, Ontario, Canada
}

\begin{abstract}
Pigeons were trained in a two-choice delayed matching-to-sample task with red and green hues. A brief postsample cue (a vertical or horizontal line) signaled whether the comparison stimuli would be presented or omitted on each trial. Comparison stimuli were always presented following the remember-cue (R-cue) trial, but never following the forget-cue (F-cue) and no-cue trials. In Experiment 1, matching accuracy on F-cue and no-cue trials was equivalent and was considerably inferior to accuracy on R-cue trials. In Experiment 2, the placement of the postsample cue was manipulated. Matching accuracy decreased as the $R$ cue was delayed in the retention interval, but performance in the F-cue condition was not affected. These data indicate that the no-cue condition can function as an implicit $F$ cue and that the $R$ cue can function to initiate and maintain rehearsal.
\end{abstract}

Delayed matching-to-sample has been extensively employed as a paradigm for studying working memory in pigeons (Maki, Moe, \& Bierley, 1977; Nelson \& Wasserman, 1978; Roberts, 1972; Roberts \& Grant, 1976). In this paradigm, the correct response is to peck the comparison stimulus, which is the same as the previously presented sample stimulus. As the length of time increases between termination of the sample stimulus and presentation of the comparison stimuli, accurate performance will increasingly depend upon the animal's ability to maintain information derived from the sample until a correct response can be made. Of course, if the comparison stimuli were not going to be presented on a particular trial, then presumably there would be no need for the animal to maintain this information. In order to assess whether pigeons are capable of selectively maintaining information in working memory, researchers have used postsample cues (e.g., a vertical or horizontal line for delayed matching-to-sample of colors) to signal whether comparisons will be omitted or presented on that trial. For example, comparison stimuli may always be omitted following a vertical line (i.e., a forget or F cue), whereas comparison stimuli are always presented following a horizontal line (i.e., a remember or $\mathbf{R}$ cue). The use of a probe testing procedure in which comparison stimuli are infrequently presented after $F$ cues has shown that $F$ cues control a lower level of matching accuracy than $\mathbf{R}$ cues, which signal presentation of the comparisons (Grant, 1981; Kendrick, Rilling, \& Stonebraker, 1981; Maki \& Hegvik, 1980; Maki, Olson, \& Rego, 1981). The mag-

This research was supported by Grant A6378 from the Natural Sciences and Engineering Research Council of Canada to Angelo Santi. The authors would like to thank Bill Reason and Colleen Hanemaayer for their technical assistance. Reprints may be obtained from Angelo Santi, Department of Psychology, Wilfrid Laurier University, Waterloo, Ontario, Canada N2L 3C5. nitude of the performance decrement produced by $F$ cues has been shown to be greater as the retention interval is lengthened (Grant, 1981; Maki \& Hegvik, 1980). Furthermore, if the occurrence of the $F$ cue is delayed within the retention interval, the effectiveness of the cue is reduced (Grant, 1981; Stonebraker \& Rilling, 1981). Experiments utilizing two cues have shown that the presentation of an $R$ cue immediately after an $F$ cue mitigates the decrement in matching accuracy produced by the $F$ cue (Grant, 1981; Stonebraker, Rilling, \& Kendrick, 1981).

Although directed forgetting effects have been most extensively studied in pigeons, they have also been obtained in studies with rats (Grant, 1982) and with squirrel monkeys (Roberts, Mazmanian, \& Kraemer, 1984). Several theoretical interpretations of the directed forgetting effect have been proposed (Grant, 1981; Maki, 1981; Rilling, Kendrick, \& Stonebraker, 1984), but the one with the greatest degree of support is the rehearsal hypothesis. This hypothesis proposes that the information derived from the sample is actively maintained in working memory by rehearsal. An F cue is believed to terminate this rehearsal process. However, whether $R$ cues function to initiate and maintain rehearsal is not entirely clear. Although experiments that present two cues in the retention interval do suggest that $R$ cues have functional control over rehearsal processes, there are contradictory data. For example, delaying an $R$ cue within a constant-length retention interval has no effect on accuracy (Grant, 1981; Stonebraker \& Rilling, 1981). In addition, similar levels of matching accuracy have been obtained on R-cue trials and on trials in which no cue is presented (i.e., no-cue trials). Grant (1981) suggested that the no-cue condition may actually function as an implicit remember cue based on the prior training history of the animal.

In the present research, we obtained direct experimental evidence regarding the functional significance of $R$ cues 
by establishing the no-cue condition as an implicit $F$ cue. This was accomplished by never presenting comparison stimuli if no cue had occurred within the retention interval. If control by an $R$ cue over matching accuracy has not been demonstrated in single cuing experiments because the absence of a cue functions as an implicit $R$ cue as a result of previous training, then in conditions in which the absence of a cue functions as an implicit $F$ cue, control over matching accuracy by an $\mathrm{R}$ cue should be demonstrable.

\section{EXPERIMENT 1}

In the first experiment reported here, pigeons were trained in a two-choice delayed matching-to-sample task with red and green hues. A brief postsample cue (a vertical or horizontal line) signaled whether the comparison stimuli would be presented or omitted on that trial. Comparison stimuli were always presented on the R-cue trials, but were never presented on the F-cue and no-cue trials during training. However, during test sessions, performance was evaluated as a function of the various cue conditions by infrequently presenting comparison stimuli on F-cue and no-cue trials. As a result, Experiment 1 provided data on whether the no-cue condition could function as an implicit $F$ cue.

\section{Method}

Subjects. Six White Carneaux pigeons, maintained at $80 \% \pm 15 \mathrm{~g}$ of their ad-lib weights and housed individually with constant access to grit and water, served as subjects. All birds had extensive experience with choice delayed matching-to-sample tasks involving color and line orientation stimuli.

Appatatus. Four Coulbourn modular operant test cages (Model E10-10), housed individually in isolation cubicles (Model E10-20), were used. Each cubicle was equipped with a ventilation fan and baffled air intake and exhaust system. Each test cage was equipped with three horizontally aligned clear plastic keys behind which projectors could display stimuli (red or green field, vertical or horizontal white line on a black background, or a black dot on a white background) onto a frosted rear projection screen (Coulbourn Model E21-18). Directly below the center keys was a $5.7 \times 5 \mathrm{~cm}$ opening that provided access to a hopper filled with mixed grain (Coulbourn Model E14-10). All experimental events and response measures were arranged and recorded by a microcomputer system located in an adjacent room.

Procedure. All birds were initially given 38 sessions of training in a delayed matching-to-sample task with cues signaling how a trial would end. Each trial began with a warning signal (a black dot on a white background) presented on the center key. A peck to this key resulted in the presentation of either a red or green sample stimulus on the center key. The sample stimulus was terminated after $5 \mathrm{sec}$ and followed by the presentation of a $1-\mathrm{sec}$ postsample cue. The postsample cue was a vertical line, a horizontal line, or no cue (i.e., the absence of any signal for $1 \mathrm{sec}$ ). For 3 birds, the vertical line signaled the occurrence of comparison stimuli $(R$ cue) and the horizontal line signaled their nonoccurrence $(F$ cue $)$. For the other 3 birds, the cue functions were reversed. For all birds, the no-cue condition signaled the nonoccurrence of comparison stimuli. Red and green comparison stimuli were presented on the side keys at the end of the retention interval only if the sample had been followed by an $R$ cue. The retention interval, as measured from the offset of the postsample cue, was initially $0 \mathrm{sec}$ and was maintained at that value until matching accuracy on R-cue trials was equal to or greater than $90 \%$ for two consecutive sessions. The retention interval was incremented by 0.5 or $1.0 \mathrm{sec}$ until accuracy was consistently maintained between $80 \%$ and $89 \%$ correct matching. During this phase of training, sessions consisted of 132 trials divided into 11 blocks of 12 trials each. Within each block of 12 trials, each combination of sample and correct side key and each of the three cue conditions occurred once in a random sequence.

Each test session consisted of 132 regular trials, which were the same as during baseline training, and an additional 12 probe trials. The 12 probe trials in each test session consisted of $4 \mathrm{R}$-cue, $4 \mathrm{~F}$ cue, and 4 no-cue trials. A single probe trial occurred randomly within each block of 12 regular trials. On probe trials, comparison stimuli were always presented, regardless of the nature of the cue presented on that trial, and correct responses were reinforced.

The probability of food reinforcement (5-sec access to mixed grain) for correct responses was manipulated in this experiment. In the differential outcome (DO) condition, reinforcement occurred with a probability of 1.0 for correct responses following one sample stimulus and a probability of 0.2 for correct responses following the other sample stimulus. In the nondifferential outcome (NDO) condition, reinforcement occurred with a probability of 0.6 following correct responses to either red or green comparison stimuli. Three birds were initially assigned to the DO condition and the other 3 to the NDO condition. Probe testing as described above was conducted for 6 sessions. The birds were then returned to baseline conditions for 3 sessions. The number of trials was decreased to 96 and an additional 16 sessions of baseline training were given, The DO birds were then switched to the NDO procedure and the NDO birds were switched to the DO procedure. Following 14 sessions of baseline training, 8 probe testing sessions were administered. On the first 2 test sessions, only 8 probe and 88 regular trials were presented, as a result of experimenter error. The remaining 6 test sessions consisted of 12 probe and 132 regular trials. The data from these 6 test sessions were combined with the data from the previous 6 test sessions so that cuing effects could be assessed for all birds under both differential and nondifferential outcome conditions. The maximum retention intervals at which the birds could maintain matching accuracy between $80 \%$ and $89 \%$ during baseline sessions varied considerably. The range of maximum delays was $0-9 \mathrm{sec}$ prior to the first test phase, with a median delay of $3 \mathrm{sec}$. The range was $0-12$ sec during the second test phase, with a median delay of $4.5 \mathrm{sec}$. However, delay interval duration was not systematically affected by the differential outcome condition to which a bird had been assigned.

\section{Results}

The mean percent of correct matching responses obtained during testing are presented in Figure 1. An analysis of variance performed on the test data included cue condition and differential outcome condition as withinsubject factors. The effect of cue condition was significant $[\mathrm{F}(2,10)=28.32, \mathrm{MSe}=54.40, \mathrm{p}<.001]$. Neither the main effect of differential outcome condition nor the interaction of cue condition $\times$ differential outcome was statistically significant [Fs < 1]. A Newman-Keuls test was performed on the mean percent correct matching as a function of cue condition. Performance in the R-cue condition was significantly higher than in the F-cue or nocue condition. Accuracy in the F-cue and the no-cue condition did not differ significantly.

\section{Discussion}

Grant (1981) found similar levels of matching accuracy on R-cue and no-cue trials. He suggested that the no-cue condition may function as an implicit $R$ cue based on the prior training history of the animal. In the present experi- 


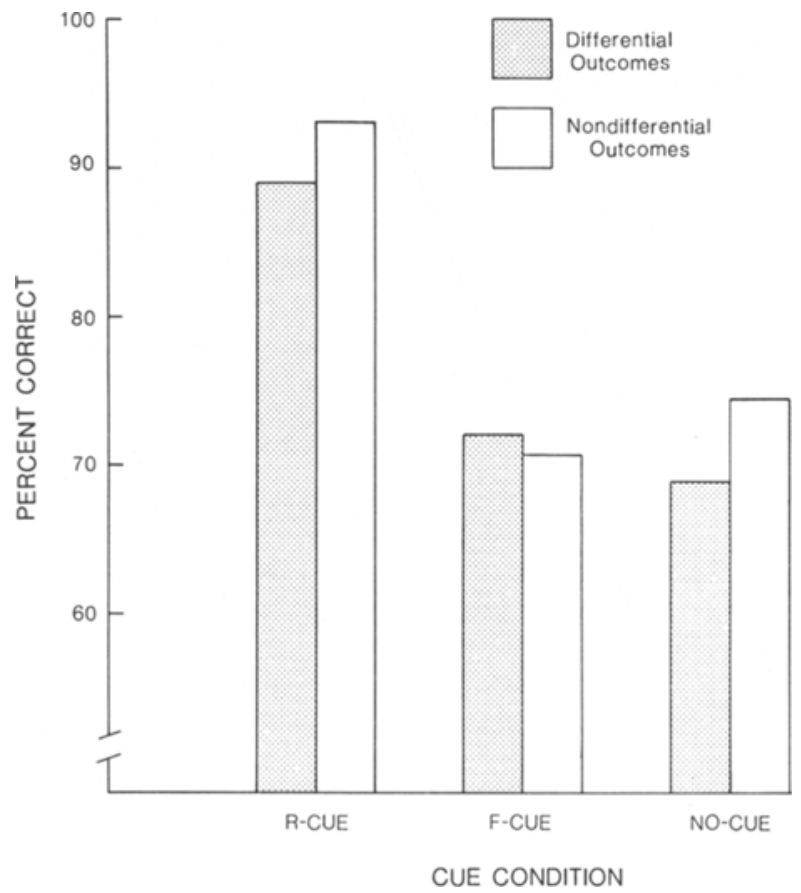

Figure 1. Mean percentage of correct responses during testing as a function of type of cue (R-cue, F-cue, or no-cue) and differential outcome condition (DO vs. NDO) in Experiment 1.

ment, matching accuracy on F-cue and no-cue trials was equivalent and was considerably inferior to that on R-cue trials. The omission of comparison stimuli on no-cue trials resulted in the no-cue condition's functioning as an implicit F cue. This supports Grant's suggestion that previous training is a critical factor determining whether the no-cue condition will act as an implicit $R$ or $F$ cue.

The magnitude of the directed forgetting effect was equivalent under both DO and NDO conditions. This suggests that the rehearsal of differential outcome expectancies may be subject to stimulus control in the same way that rehearsal of sample stimulus attributes or response instructions with regard to comparison stimuli are. However, no firm conclusion about this is possible, because differential outcome conditions did not systematically affect the length of the delay interval necessary to maintain accuracy between $80 \%$ and $89 \%$ during baseline sessions. In studies by DeLong and Wasserman (1981) and Santi and Roberts (1985a, 1985b), differential probability of reinforcement produced an enhanced acquisition of discrimination and superior memory performance in pigeons. The failure to obtain an effect of differential outcomes in the present study may be due to the concomitant use of a directed forgetting procedure or to the lack of sufficient training under differential outcome conditions.

Whatever the nature of the memorial representation supporting performance in the present experiment, compelling evidence for the stimulus control of rehearsal could be obtained by manipulating the temporal location of the
$R$ cue and the $F$ cue within the retention interval. We undertook the second experiment with this in mind.

\section{EXPERIMENT 2}

An $\mathrm{F}$ cue is assumed to block or terminate the active maintenance of information represented during the retention interval. This assumption is supported by the finding that the effectiveness of the $\mathrm{F}$ cue is reduced if it occurs later in the retention interval (Grant, 1981; Stonebraker \& Rilling, 1981). When a single cue has been presented in the retention interval, delaying the $R$ cue has had no effect on accuracy (Grant, 1981; Stonebraker \& Rilling, 1981). Presumably, this is due to the pigeons' engaging in rehearsal even in the absence of an $R$ cue. Given that our Experiment 1 found that the no-cue condition could function as an implicit $\mathrm{F}$ cue, it should be possible to directly assess whether the R cue could function to initiate and maintain rehearsal by manipulating the temporal location of the cue. In Experiment 2, we expected to obtain results that would be the reciprocal of those previously reported by Grant (1981) and Stonebraker and Rilling (1981). That is, performance on F-cue trials should not be affected by location of the F cue in the retention interval, whereas performance on R-cue trials should decrease as the $R$ cue was delayed within the retention interval. As in Experiment 1, testing was conducted with both DO and NDO conditions for all subjects.

\section{Method}

Subjects and Apparatus. The subjects and apparatus were the same as those used in Experiment 1.

Procedure. All birds were given 10 sessions of training, which were the same as those which preceded testing in Experiment 1 except that all birds were assigned to the NDO condition. One of the birds was terminated from the study because performance at or above $80 \%$ correct could not be maintained at delays greater than $1 \mathrm{sec}$. A minimum delay of $3 \mathrm{sec}$, measured from offset of the postsample cue, was required, so that cue placement could be meaningfully varied. The remaining 5 birds maintained performance at or above $80 \%$ correct at retention intervals that varied between birds $(3,4,5,10$, and $12 \mathrm{sec})$. After the 10 training sessions, we administered cue-position testing under NDO conditions. Following this testing, all birds were returned to baseline NDO conditions for 5 sessions. Then all birds were switched to DO condition, in which the reinforcement probability assignments were the same as each bird had received in Experiment 1. Following 10 sessions of training under DO conditions, we administered cue-position testing.

Cue-position testing was administered in the same way for DO and NDO conditions. Each test session alternated with a baseline session in which no probe trials occurred. This was done to minimize any disruption which could occur as a result of probe testing at various cue positions. Each baseline session consisted of 96 trials (32 R-cue, 32 F-cue, and 32 no-cue trials). Each test session consisted of 96 trials and an additional 12 probe trials ( 4 R-cue, 4 F-cue, and 4 no-cue trials). Within each test session, trials were organized into 12 blocks of 9 trials each ( 1 probe and 8 regular trials). Comparison stimuli were presented at the end of the retention interval on all probe trials, and correct responses were reinforced according to the probabilities defined for the DO and NDO conditions. On regular trials, $R$ and $F$ cues were always presented at the beginning of the retention interval. On probe trials, $R$ and $F$ cues were 
presented at the beginning, the middle, or the end of the retention interval. Cue position was manipulated between sessions and randomized within blocks of three test sessions. As in the previous experiment, cue duration was $1 \mathrm{sec}$. In the case of the no-cue condition, this functionally added $1 \mathrm{sec}$ to the retention interval. Twelve test sessions were administered in each of the two test phases.

\section{Results}

Test performance as a function of cue, cue position, and differential outcome condition are presented in Figure 2. For purposes of data analysis, the no-cue condition was treated as if the temporal location of the no cue was the same as that used for $R$ and $F$ cues in each test session. No effects due to the differential outcome manipulation were obtained. However, the analysis of variance did reveal a significant effect of cue condition $[\mathrm{F}(2,8)=8.59, \mathrm{MSe}=104.02, \mathrm{p}<.05]$ and cue position $[\mathrm{F}(2,8)=8.72, \mathrm{MSe}=89.90, \mathrm{p}<.01]$ and a cue condition $\times$ cue position interaction $[F(4,16)=8.36$, $\mathrm{MSe}=100.49, \mathrm{p}<.001]$. A simple main effect analysis of this interaction indicated that there was an effect of cue position on R-cue trials $[F(2,8)=14.68]$, but not on F-cue or no-cue trials (Fs $<2$ ). A Newman-Keuls test confirmed that accuracy was significantly less when the $R$ cue was presented in the middle or at the end of the retention interval than when the $R$ cue was presented at the beginning of the retention interval. The simple main effect analysis also revealed that there was an effect of cue condition when the cues were presented at the beginning of the retention interval $[\mathrm{F}(2,8)=25.95]$, but not when they were presented in the middle or at the end of the retention interval $(\mathrm{Fs}<1)$.

\section{Discussion}

When the $\mathrm{R}$ or the $\mathrm{F}$ cue was presented at the beginning of the retention interval, the results replicated those obtained in Experiment 1. Matching accuracy was high on R-cue trials and low on F-cue and no-cue trials. The equivalent performance on F-cue and no-cue trials indicated that the absence of a cue continued to function as an implicit $F$ cue. The effectiveness of the $R$ cue in maintaining high levels of matching accuracy was reduced when it was presented later in the retention interval. These results indicate that the $\mathrm{R}$ cue can function to initiate and maintain rehearsal, but that it cannot function effectively as a retrieval cue for information that is not being actively rehearsed. When the $\mathrm{R}$ cue was presented in the middle or at the end of the retention interval, it did not control a level of matching accuracy significantly above that controlled by the F-cue or no-cue condition.

\section{GENERAL DISCUSSION}

The present experiments provide further support for the hypothesis that pigeons actively maintain or rehearse information derived from the sample stimulus during the retention interval. In previous studies involving variation in cue placement and comparison of R-cue, F-cue, and no-cue trials, it was found that $F$ cues terminated or reduced rehearsal but that $R$ cues had little effect on performance relative to no-cue conditions (Grant, 1981; Stonebraker \& Rilling, 1981). The present results indicate that if the no-cue condition functions as an implicit $\mathrm{F}$ cue, then the R-cue condition provides data consistent
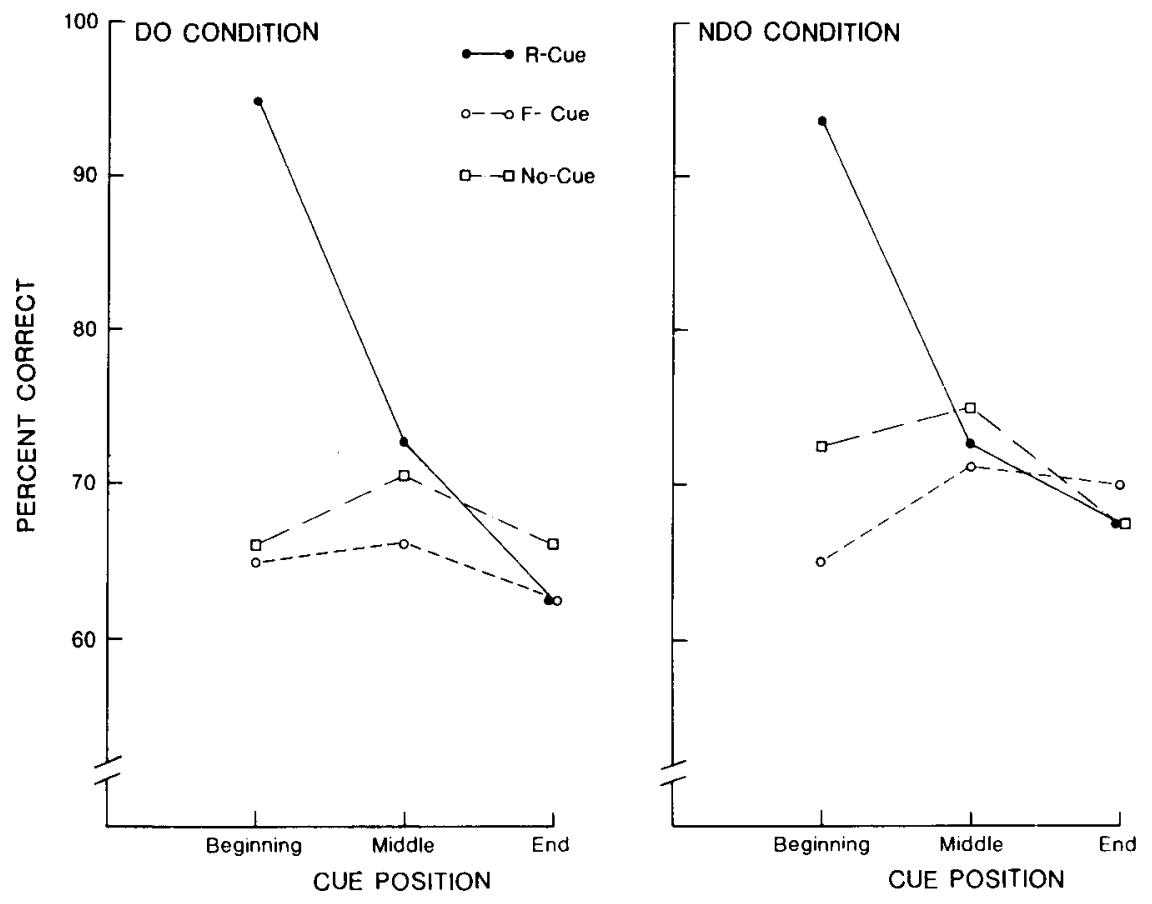

Figure 2. Mean percentage of correct responses during testing as a function of cue position (beginning, middle, or end), type of cue (R-cue, F-cue, or no-cue) and differential outcome condition (DO vs. NDO) in Experiment 2. 
with the hypothesis that $R$ cues can initiate and maintain rehearsal. This conclusion is compatible when that derived from studies in which $\mathrm{R}$ cues attenuate the effectiveness of a previously presented F cue (Grant, 1981; Stonebraker et al., 1981). In addition, the results of Experiment 2 are consistent with results obtained when the presentation of the $\mathrm{R}$ cue is delayed following the prior presentation of an F cue. Stonebraker et al. (1981) showed that the effectiveness with which the $R$ cue attenuates an F-cue effect diminishes as the $\mathrm{R}$ cue is delayed within the retention interval.

Grant (1984) studied directed forgetting effects in an intratrial interference procedure in which two samples were presented successively on each trial and each sample was followed by a postsample $\mathrm{R}$ or $\mathrm{F}$ cue. He found that presentation of an $\mathrm{F}$ cue after an interfering sample increased accuracy relative to baseline trials, whereas presentation of an $\mathrm{F}$ cue after the target sample decreased accuracy relative to baseline trials. In addition, the ability of the $F$ cue to increase accuracy when presented after an interfering sample was enhanced as the temporal interval between the two successively presented samples was increased. These data provide strong support for an explanation of directed forgetting effects in terms of rehearsal. An F cue terminates rehearsal processes, whereas an $\mathrm{R}$ cue can initiate and maintain rehearsal processes. The results of Experiment 2 in the present study also in dicate that an $\mathrm{R}$ cue cannot serve as a retrieval cue for information that is not being actively rehearsed. This was evident in the failure of the $R$ cue to control levels of matching accuracy significantly greater than those controlled by the F-cue or no-cue condition when the $\mathrm{R}$ cue was presented in the middle or at the end of the retention interval.

In both experiments, directed forgetting effects were obtained with a DO procedure as well as with an NDO procedure. However, the significance of this finding is difficult to assess, because there was no clear evidence of a differential outcome effect in the present data. Previous investigators have shown that differential outcomes enhance memory performance by establishing outcome expectancies that mediate responding to comparison stimuli (Edwards, Jagielo, Zentall, \& Hogan, 1982; Honig, Matheson, \& Dodd, 1984; Peterson, 1984). If a differential outcome effect could be demonstrated with a directed forgetting procedure, then it could be determined whether outcome expectancies are just as sensitive to directed forgetting manipulations as other types of representations. This would provide evidence for the claim by Rilling et al. (1984) that the analysis of rehearsal as an active maintenance of representations does not need to be concerned with the nature of the representation being rehearsed. mance in pigeons. Journal of Experimental Psychology: Animal Behavior Processses, 7, 394-412.

Edwards, C. A., Jagielo, J. A., Zentall, T. R, \& Hogan, D. E. (1982). Acquired equivalence and distinctiveness in matching to sample by pigeons: Mediation by reinforcer-specific expectancies. Journal of Experimental Psychology: Animal Behavior Processes, 8, 244-259.

GRANT, D. S. (1981). Stimulus control of information processing in pigeon short-term memory. Learning \& Motivation, 12, 19-39.

GRANT, D. S. (1982). Stimulus control of information processing in rat short-term memory. Joumal of Experimental Psychology: Animal Behavior Processes, 8, 154-164.

Grant, D. S. (1984). Directed forgetting and intratrial interference in pigeon delayed matching. Canadian Journal of Psychology, 38, 166-177.

Honig, W. K., Matheson, W. R., \& Dodd, P. W. D. (1984). Outcome expectancies as mediators for discriminative responding. Canadian Journal of Psychology, 38, 196-217.

Kendrick, D. F., Rilling, M., \& Stonebraker, T. B. (1981). Stimulus control of delayed matching in pigeons: Directed forgetting. Journal of the Experimental Analysis of Behavior, 36, 241-251.

MAKI, W. S. (1981). Directed forgetting in animals. In N. E. Spear \& R. R. Miller (Eds.), Information processing in animals: Memory mechanisms (pp. 199-226). Hillsdale, NJ: Erlbaum.

MAKI, W. S., \& HeGviK, D. K. (1980). Directed forgetting in pigeons. Animal Learning \& Behavior, 8, 567-574.

Maki, W. S., Moe, J. C., \& Bierley, C. M. (1977). Short-term memory for stimuli, responses and reinforcers. Journal of Experimental Psychology: Animal Behavior Processes, 3, 156-177.

MAKI, W. S., Olson, D., \& REgo, S. (1981). Directed forgetting in pigeons: Analysis of cue functions. Animal Learning \& Behavior, 9 , 189-195.

Nelson, K. R., \& Wasserman, E. A. (1978). Temporal factors influencing the pigeon's successive matching-to-sample performance: Sample duration, intertrial interval and retention interval. Journal of the Experimental Analysis of Behavior, 30, 153-162.

Peterson, G. B. (1984). How expectancies guide behavior. In H. L. Roitblat, T. G. Bever, H. S. Terrace (Eds.), Animal cognition (pp. 135-148). Hillsdale, NJ: Erlbaum.

Riluing, M., Kendrick, D. F., \& Stonebraker, T. B. (1984). Directed forgetting in context. In G. H. Bower (Ed.), The psychology of learning and motivation: Advances in research and theory (Vol. 18, pp. 175198). New York: Academic Press.

Roberts, W. A. (1972). Short-term memory in the pigeon: Effects of repetition and spacing. Joumal of Experimental Psychology, 94, 74-83.

RoBERTS, W. A., \& GRANT, D. S. (1976). Studies of short-term memory in the pigeon using the delayed matching-to-sample procedure. In D. L. Medin, W. A. Roberts, R. T. Davis (Eds.), Processes of animal memory (pp. 79-112). Hillsdale, NJ: Erlbaum.

Roberts, W. A., Mazmanian, D. S., \& Kraemer, P. J. (1984). Directed forgetting in monkeys. Animal Learning \& Behavior, 12, $29-40$

SANTI, A., \& RoberTs, W. A. (1985a). Prospective representation: The effects of varied mapping of sample stimuli to comparison stimuli and differential trial outcomes on pigeons' working memory. Animal Learning \& Behavior, 13, 103-108.

SANTI, A., \& ROBERTS, W. A. (1985b). Reinforcement expectancy and trial spacing effects in delayed matching-to-sample by pigeons. Animal Learning \& Behavior, 13, 274-284.

Stonebraker, T. B., \& Rilling, M. (1981). Control of delayed matching-to-sample performance using directed forgetting techniques. Animal Learning \& Behavior, 9, 196-201.

Stonebraker, T. B., Rilling, M., \& Kendrick, D. F. (1981). Time dependent effects of double-cuing in directed forgetting. Animal Learning \& Behavior, 9, 385-394.

\section{REFERENCES}

DeLong, R. E., \& Wasserman, E. A. (1981). Effects of differential reinforcement expectancies on successive matching-to-sample perfor-
(Manuscript received June 6, 1985; revision accepted for publication October 18, 1985.) 